\title{
Sustained Efficacy of Cetuximab after Therapy Discontinuation in a Patient with Advanced Colorectal Cancer
}

\author{
Gerardo Rosati ${ }^{1}$, Enrico Scarano ${ }^{2}$, Antonio Avallone ${ }^{3}$ and Domenico Bilancia ${ }^{1}$ \\ ${ }^{1}$ Medical Oncology Unit, "S. Carlo" Hospital of Potenza, Italy \\ ${ }^{2}$ Radiology Unit, "S. Carlo" Hospital of Potenza, Italy \\ ${ }^{3}$ Department of Gastrointestinal Medical Oncology, National Cancer Institute of Naples, Italy
}

Corresponding author: Dr Gerardo Rosati, Medical Oncology Unit , “S. Carlo" Hospital of Potenza, Via Sanremo 197, 85100 Potenza, Italy, Tel: +39-0971-612273; Fax: +39-0971-613000; E-mail: oncogerry@yahoo.it

Received date: Jan 04, 2014, Accepted date: Mar 28, 2014, Published date: May 07, 2014

Copyright: (c) 2014 Rosati G, et al. This is an open-access article distributed under the terms of the Creative Commons Attribution License, which permits unrestricted use, distribution, and reproduction in any medium, provided the original author and source are credited

\begin{abstract}
In pivotal phase II and III studies, the targeting monoclonal antibody cetuximab has been shown to improve the efficacy of standard chemotherapy regimens used in first-line treatment of metastatic colorectal cancer. Safety data analyses from these studies indicate that cetuximab plus chemotherapy appears to be associated with a higher incidence of grade 3-4 adverse events than chemotherapy alone. In particular, dermatological toxicity and skin reactions can require an early discontinuation therapy affecting clinical outcomes. Herein, we present one case which document a sustained long-lasting efficacy of cetuximab triggered by a presumable immune response after discontinuation of therapy, in order to focus on the possibility to suggest a different use of antibody.
\end{abstract}

Keywords: Cetuximab; Colorectal cancer; Immune response

\section{Introduction}

The epidermal growth factor receptor (EGFR)-targeting monoclonal antibody, cetuximab, has been shown to improve the efficacy of standard chemotherapy regimens used in the first-line treatment of several common cancers, including metastatic colorectal cancer (mCRC) [1-3]. In particular, the CRYSTAL (Cetuximab Combined with Irinotecan in First-Line Therapy for Metastatic Colorectal Cancer) study met its primary end point in demonstrating that the addition of cetuximab to a combined first-line chemotherapy regimen of irinotecan, infusional fluorouracil, and leucovorin (FOLFIRI) statistically significantly reduced the risk of progression of mCRC compared with chemotherapy alone (hazard ratio $0.85 ; \mathrm{P}=$. 048) [1]. Successively, an updated analysis according to tumour KRAS and BRAF mutation status and with increased follow-up time has also demonstrated unequivocally an improvement of overall survival in patients whose tumours are wild-type for KRAS [4]. In addition, a randomized phase II study has confirmed that, when combined with oxaliplatin-based chemotherapy (FOLFOX), cetuximab increased the overall response rate (ORR) and reduced the risk of progression in patients whose tumours were wild-type for KRAS if compared with those treated with chemotherapy alone [5]. However, cetuximab therapy integration into protocols has been hampered by a fair number of grade $3 / 4$ toxicities varies between $18 \%$ and $19.5 \%$ for skin reactions, $28.2 \%$ and $30 \%$ for neutropenia, and $11 \%$ and $15.7 \%$ for diarrhoea [4,5]. Although recent data from the CRYSTAL trial suggest that skin reactions related to anti-EGFR therapy do not impact on patient quality of life [6], skin toxicities may have negative psychosocial impact and sometimes affect clinical outcomes causing discontinuation of therapy. Herein we report an unusual case of a man with liver metastases from CRC diagnosed in our institution, treated with FOLFOX plus cetuximab, and with sustained radiological response after discontinuation of therapy due to unacceptable skin toxicity.

\section{Case Report}

A 69-year-old retired man came to our attention in February 2012. His history reported a total laryngectomy with neck dissection for a cancer of the larynx at approximately 52 years of age. A permanent tracheostomy was fashioned and postoperative irradiation was required. This combined treatment ensured the recovery and subsequent follow-up showed no recurrence of the disease. One month earlier, in January 2012, he had undergone a left hemicolectomy resection after diagnosis of adenocarcinoma of the descending colon. An abdominal magnetic resonance imaging (MRI) revealed the presence of multiple hepatic nodules, the largest of approximately $4 \mathrm{~cm}$ in longest diameter at the level of the IV segment (Figure 1A). CT scans with contrast failed to show any evidence of further metastases. Biopsy of the liver confirmed the diagnosis of metastasis of colon adenocarcinoma. After the execution of the KRAS test, the tumour was wild-type at codons 12 and 13 of the corresponding gene. Serum chemistry analysis showed bilirubin 0.4 $\mathrm{mg} / \mathrm{dl}, \gamma$-glutamyltransferase (GGT) $38 \mathrm{U} / \mathrm{l}$, alkaline phosphatase (AP) $110 \mathrm{U} / \mathrm{l}$, AST $26 \mathrm{U} / \mathrm{l}$, ALT $28 \mathrm{U} / \mathrm{l}$ and cholinesterase $6245 \mathrm{U} / \mathrm{l}$. Other laboratory test results were normal. At this point, on day 1 of a 14-day treatment cycle, the patient received cetuximab (initial dose 400 $\mathrm{mg} / \mathrm{m}^{2}$ infused over 2 hours, and $250 \mathrm{mg} / \mathrm{m}^{2}$ weekly, over 1 hour, thereafter) followed after 1 hour by FOLFOX6 (leucovorin $100 \mathrm{mg} / \mathrm{m}^{2}$ followed by 5 -fluorouracil bolus $400 \mathrm{mg} / \mathrm{m}^{2}$ and 46 -hour infusion $2400 \mathrm{mg} / \mathrm{m}^{2}$ with oxaliplatin $100 \mathrm{mg} / \mathrm{m}^{2}$ ), until disease progression or the occurrence of unacceptable toxicity. No haematological side effects occurred during the first 8 weeks of therapy while a grade 2 maculopapular rash appeared on the face. It was sufficiently controlled by the application of a cream containing vitamin $K_{1}$. A reduced diameter of liver metastases (nodule of $28 \mathrm{~mm}$ at the level of the IV segment) was noted on abdomen MRI (Figure 1B), and we determined 
Citation: Rosati G, Scarano E, Avallone A, Bilancia D (2014) Sustained Efficacy of Cetuximab after Therapy Discontinuation in a Patient with Advanced Colorectal Cancer . J Carcinog Mutagen S10: 004. doi:10.4172/2157-2518.S10-004

Page 2 of 3

the therapy effect to be a partial response. Successively, the combined cetuximab-based therapy was regularly continued weekly until August. The patient had experienced only mild anaemia of grade 1 , but it seemed much worse skin toxicity. The maculopapular rash was managed not only with the application of the vitamin $\mathrm{K}_{1}$ cream, but also with the oral assumption of tetracycline and topical steroid treatment. At the start of cycle 7 of combined therapy, the skin toxicity was aggravated by the appearance of a paronychia of fingers, dry skin, and skin fissures. Although of grade 1, paronychia and skin fissures showed annoying and painful requiring the contribution of ointment with polyethylene glycols and allantoin whereas a moisturizer restored partially the permeability barrier and treated dry skin. After the tenth cycle of therapy, administered in August, a grade 4 paronychia with severe impairment of quality of life led to discontinuation of any drug. A further reduction in the diameter of liver metastases (nodule of 19 $\mathrm{mm}$ at the level of the IV segment) was confirmed by an MRI (Figure 1C). After about two months, the skin toxicity appeared resolved whereas a subsequent MRI performed in November showed a surprising further reduction of the size and number of hepatic metastases in the absence of any treatment (Figure 1D). In the following months, until October 2013, were not observed changes nor in the patient's clinical condition or in the radiological patterns of disease (Figures $1 \mathrm{E}$ and $1 \mathrm{~F}$ ).
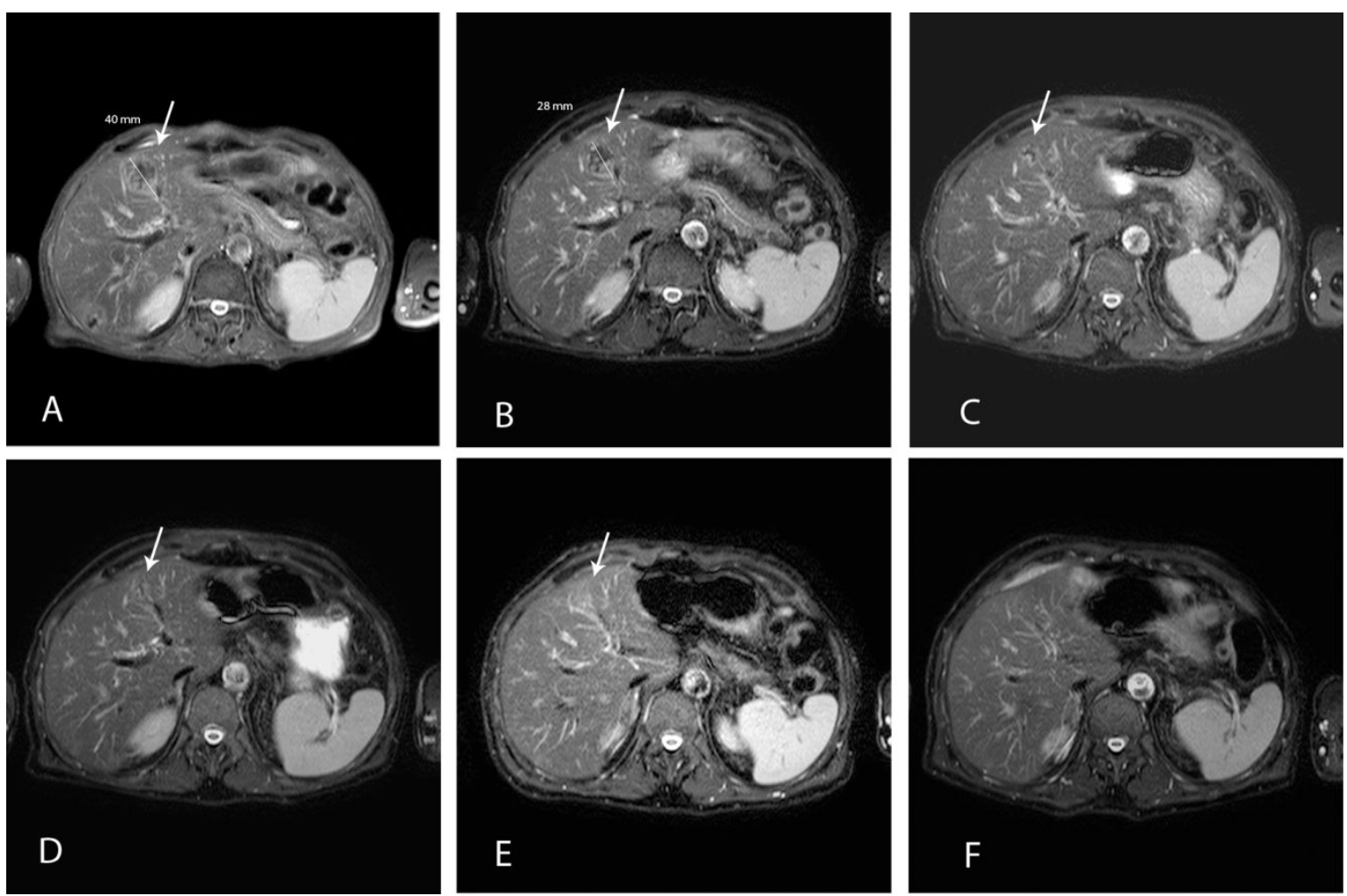

Figure 1: MRI performed sequentially in February 2012 (1A), May 2012 (1B), August 2012 (1C), November 2012 (1D), March 2013 (1E), and October $2013(1 \mathrm{~F})$.

\section{Discussion}

Although the advantages in terms of ORR, progression-free survival (PFS), and overall survival (OS) are more straightforward with the combination with irinotecan than that with oxaliplatin, patients whose tumours are wild-type for KRAS still have a statistically significant increased chance of response $(61 \%$ v $37 \% ; \mathrm{P}=.011)$ and a lower risk of disease progression (hazard ratio $=0.57 ; \mathrm{P}=.0163$ ) if treated with cetuximab with FOLFOX rather than with FOLFOX alone. Moreover, in the OPUS study, the median duration of response was also longer in the cetuximab group (9.0 v 5.7 months) [5]. Nevertheless, even these patients, like all the others, show resistance to the therapy and inevitably fall in clinical progression. This has not happened yet for our patient, although the treatment has been suspended after ten cycles in August of 2012 for unacceptable skin toxicity. To date, after 20 months from started therapy, the patient remains with a partial remission of disease. How to explain this unusual and extraordinary result? The EGFR is frequently overexpressed or abnormally activated in tumours. Early studies with anti-EGFR monoclonal antibody $(\mathrm{mAb})$ were shown to inhibit the growth of cancer cells bearing EGFR [7]. Cetuximab is a recombinant human-murine chimeric mAb targeted specifically to the external domain of EGFR, compete with the ligand binding, and block activation of receptor tyrosine kinase by EGF or transforming growth factor alpha. In addition, cetuximab induces antibody-mediated receptor dimerization, resulting in receptor downregulation, and this effect may be important for its growthinhibitory capacity [8]. As result of these effects, cetuximab interferes with a number of key cellular functions regulated by the receptor: cellcycle arrest, potentiation of apoptosis, inhibition of angiogenesis, inhibition of tumour-cell invasion and metastasis, and augmentation of antitumour effects of chemotherapy and radiotherapy. However, cetuximab can also elicit likely immunological events that occur in treated patients: antibody-dependent cellular cytotoxicity (ADCC), cross talk among immune cells including natural killer (NK) cells and 
Citation: Rosati G, Scarano E, Avallone A, Bilancia D (2014) Sustained Efficacy of Cetuximab after Therapy Discontinuation in a Patient with Advanced Colorectal Cancer . J Carcinog Mutagen S10: 004. doi:10.4172/2157-2518.S10-004

Page 3 of 3

dendritic cells (DC), and generation of tumour antigen-specific $\mathrm{T}$ lymphocyte responses (Figure 2) [9]. The ADCC activity depends on the degree of EGFR expression on tumour cell surfaces independently of EGFR mutation status [10]. The DC and NK cells interaction, facilitating their recruitment to the tumour site, can modulate both the innate immune response in the local tumour microenvironment and the adaptive immune response in secondary lymphoid organs. The generation of these events may offer substantial advantages in response to the treatment of cancer patients. MAb can generate a longlasting immunological central memory which can justify oneself a prolonged clinical benefit in some patients, as in our case. This is not true for all patients, but undoubtedly the knowledge of this act ion modality of cetuximab should be thorough and could be synergized with other forms of immunotherapy. On the other hand, Kurai et al. have demonstrated that ADCC activity was enhanced by interleukin-2 through activation of NK cells [11]. In this scenario, we believe that could be not necessary a continuous administration of cetuximab combined to chemotherapy and further clinical studies should be planned to investigate our hypothesis in the treatment of wild-type RAS mCRC patients.

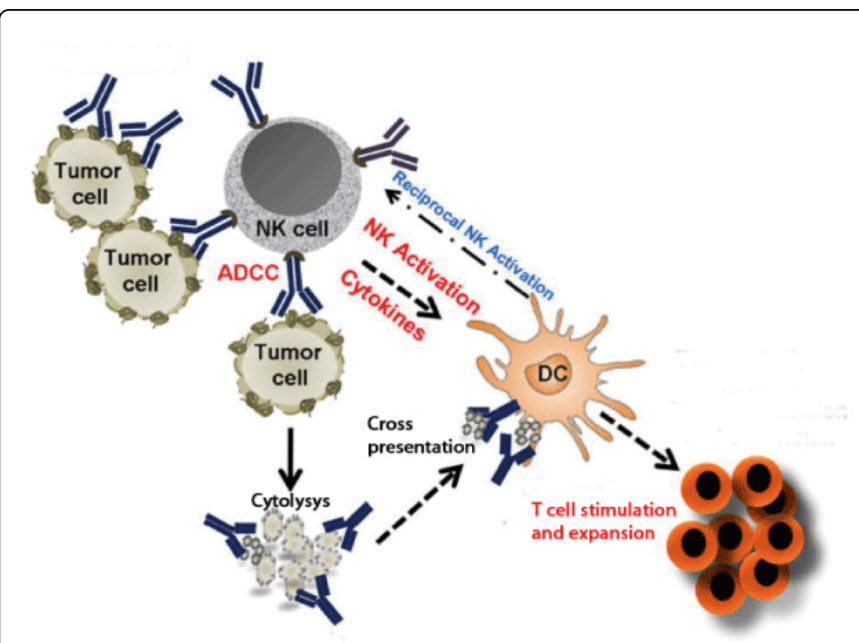

Figure 2: Cellular cascade leading to antitumour immune response generated by cetuximab.

\section{References}

1. Van Cutsem E, Köhne CH, Hitre E, Zaluski J, Chang Chien CR, et al. (2009) Cetuximab and chemotherapy as initial treatment for metastatic colorectal cancer. N Engl J Med 360: 1408-1417.

2. Vermorken JB, Mesia R, Rivera F, Remenar E, Kawecki A, et al. (2008) Platinum-based chemotherapy plus cetuximab in head and neck cancer. N Engl J Med 359: 1116-1127.

3. Pirker R, Pereira JR, Szczesna A, von Pawel J, Krzakowski M, et al. (2009) Cetuximab plus chemotherapy in patients with advanced non-small-cell lung cancer (FLEX): an open-label randomised phase III trial. Lancet 373: 1525-1531.

4. Van Cutsem E, Köhne CH, Láng I, Folprecht G, Nowacki MP, et al. (2011) Cetuximab plus irinotecan, fluorouracil, and leucovorin as firstline treatment for metastatic colorectal cancer: updated analysis of overall survival according to tumor KRAS and BRAF mutation status. J Clin Oncol 29: 2011-2019.

5. Bokemeyer C, Bondarenko I, Makhson A, Hartmann JT, Aparicio J, et al. (2009) Fluorouracil, leucovorin, and oxaliplatin with and without cetuximab in the first-line treatment of metastatic colorectal cancer. J Clin Oncol 27: 663-671.

6. Láng I, Köhne CH, Folprecht G, Rougier P, Curran D, et al. (2013) Quality of life analysis in patients with KRAS wild-type metastatic colorectal cancer treated first-line with cetuximab plus irinotecan, fluorouracil and leucovorin. Eur J Cancer 49: 439-448.

7. Mendelsohn J, Baselga J (2003) Status of epidermal growth factor receptor antagonists in the biology and treatment of cancer. J Clin Oncol 21: 2787-2799.

8. Fan Z, Lu Y, Wu X, Mendelsohn J (1994) Antibody-induced epidermal growth factor receptor dimerization mediates inhibition of autocrine proliferation of A431 squamous carcinoma cells. J Biol Chem 269: 27595-27602.

9. Lee SC, Srivastava RM, López-Albaitero A, Ferrone S, Ferris RL (2011) Natural killer (NK): dendritic cell (DC) cross talk induced by therapeutic monoclonal antibody triggers tumor antigen-specific $\mathrm{T}$ cell immunity. Immunol Res 50: 248-254.

10. Kimura H, Sakai K, Arao T, Shimoyama T, Tamura T, et al. (2007) Antibody-dependent cellular cytotoxicity of cetuximab against tumor cells with wild-type or mutant epidermal growth factor receptor. Cancer Sci 98: 1275-1280.

11. Kurai J, Chikumi H, Hashimoto K, Yamaguchi K, Yamasaki A, et al (2007) Antibody-dependent cellular cytotoxicity mediated by cetuximab against lung cancer cell lines. Clin Cancer Res 13: 1552-1561. 